Esen Hanaç*

\title{
On the evolution of solutions of Burgers equation on the positive quarter-plane
}

https://doi.org/10.1515/dema-2019-0020

Received December 9, 2018; accepted May 14, 2019

Abstract: In this paper we investigate an initial-boundary value problem for the Burgers equation on the positive quarter-plane;

$$
\begin{aligned}
v_{t}+v v_{x}-v_{x x} & =0, \quad x>0, \quad t>0, \\
v(x, 0) & =u_{+}, \quad x>0, \\
v(0, t) & =u_{b}, \quad t>0
\end{aligned}
$$

where $x$ and $t$ represent distance and time, respectively, and $u_{+}$is an initial condition, $u_{b}$ is a boundary condition which are constants $\left(u_{+} \neq u_{b}\right)$. Analytic solution of above problem is solved depending on parameters $\left(u_{+}\right.$and $u_{b}$ ) then compared with numerical solutions to show there is a good agreement with each solutions.

Keywords: Burgers equation, traveling wave solution (TW), numeric solutions

MSC: $65 \mathrm{MXX}$

\section{Introduction}

An initial and boundary value problem for Burgers' equation on the positive quarter-plane will be examined in this section. The distinct equation to be discussed is given by

$$
\begin{aligned}
v_{t}+v v_{x}-v_{x x} & =0, \quad x>0, \quad t>0, \\
v(x, 0) & =u_{+}, \quad x>0, \\
v(0, t) & =u_{b}, \quad t>0,
\end{aligned}
$$

and $u_{+}$is an initial condition and $u_{b}$ is a boundary condition $\left(u_{b} \neq u_{+}\right)$which are both integers.

Later on, the initial-boundary value problem (1)-(3) is labeled as QP, the stationary solution as SS, expansive wave as EW and wave speed as ws. The method in [7-9] is used to set the schema of the solution to QP. The problem parameters $u_{+}$and $u_{b}$ play a vital role for the large t-solution of QP. In particular, the attractor of the solution to QP is given by:

(i) TW with positive ws while $-u_{b}<u_{+}<u_{b}$ with $u_{b}>0$.

(ii) SS when $\left(-u_{+}>u_{b}>u_{+}\right.$with $\left.u_{+}<0\right)$ or when $0 \geqslant u_{+}>u_{b}$.

(iii) The schema containing a conjunction of a EW and SS while $u_{b}<0$ and $u_{+}>0$.

(iv) An EW when $u_{+}>u_{b} \geqslant 0$.

The methodology in [7] (see [6] as well) has been applied to complete the large t asymptotic schema of QP. We start with the case (i) where $-u_{b}<u_{+}<u_{b}$ with $u_{b}>0$.

^Corresponding Author: Esen Hanaç: Department of Mathematics, Adıyaman University, Turkey;

E-mail: ehanac@adiyaman.edu.tr 


\section{Asymptotic schema while $-\mathbf{u}_{\mathrm{b}}<\mathbf{u}_{+}<\mathbf{u}_{\mathrm{b}}, \mathbf{u}_{\mathrm{b}}>\mathbf{0}$}

We start with the first which we denote as $I^{\star}$, in this region

$$
v(\gamma, t)=\left(\left(u_{b}-u_{+}\right) \operatorname{erfc}\left(\frac{\gamma}{2}\right)+u_{+}\right)+o(1),
$$

as $t \rightarrow 0$ with $\gamma=O(1)(0 \leqslant \gamma<\infty)$. In the region $\mathrm{II}^{\star}$,

$$
v(x, t)=u_{+}+\exp \left(-\frac{x^{2}}{4 t}+\frac{1}{2} \ln t+\frac{u_{+}}{2} x-\ln x+\ln \mathcal{A}^{\star}+o(1)\right)
$$

as $t \rightarrow 0$ with $x=O(1)(>0)$, and where $\mathcal{A}^{\star}=\frac{2}{\sqrt{\pi}}\left(u_{b}-u_{+}\right)(>0)$. The solution of QP as $t \rightarrow 0$ is solved asymptotically and now completed with equations (4) and (5) bringing an irreversible resemblance to the QP as $t \rightarrow 0$. The appearance of equation (5) of region $\mathrm{II}^{\star}$ for $x \gg 1$ as $t \rightarrow 0$ requires a new region that is labeled as region $\mathrm{III}^{\star}$, region $\mathrm{III}^{\star}$

$$
v(x, t)=u_{+}+\exp \left(-\frac{x^{2}}{4 t}+\frac{u_{+}}{2} x-\ln x+\left(\frac{1}{2} \ln t-\frac{u_{+}^{2}}{4} t+\ln \mathcal{A}^{\star}\right)+o(1)\right)
$$

as $x \rightarrow \infty$ with $t=O(1)(>0)$. As $t \rightarrow \infty$, the equation (6) of region III ${ }^{\star}$ goes on to stay irreversible for $x \gg t$. Nevertheless, while $x=O(t)$ it becomes reversible. To work on a new region IV* we need to introduce a new coordinate $y=\frac{X}{t}$ where $y=O(1)$ as $t \rightarrow \infty$ and seek an equation of the appearance (as suggested by (6))

$$
\begin{gathered}
v(y, t)=u_{+}+e^{-N N(y, t)} \quad \text { as } \quad t \rightarrow \infty, \\
N N(y, t)=n_{0}^{\star}(y) t+n_{1}^{\star}(y) \ln t+n_{2}^{\star}(y)+o(1),
\end{gathered}
$$

where $y=O(1)(>0)$ as $t \rightarrow \infty$ and $n_{0}^{\star}(y)>0, n_{1}^{\star}(y)$ and $n_{2}^{\star}(y)$ are functions to be identified. On substituting (7) and (8) into equation (1) and after some computations a one-parameter family of linear solutions has occurred,

$$
n_{0}^{\star}(y)=A^{+}\left[y-\left(A^{+}+u_{+}\right)\right], \quad y>0
$$

for any $A^{+} \in \mathbb{R}$, together with the associated envelope solution

$$
n_{0}^{\star}(y)=\frac{\left(y-u_{+}\right)^{2}}{4}, \quad y>u_{+} .
$$

Combining of (9) and (10) which stay continuous and differentiable also provides an envelope touching solutions. The solution (9)-(10) is given either by the envelope solution

$$
n_{0}^{\star}(y)=\frac{\left(y-u_{+}\right)^{2}}{4}, \quad y>u_{+}
$$

or by the family of envelope touching solutions

$$
n_{0}^{\star}(y)=\left\{\begin{array}{l}
\frac{\left(y-u_{+}\right)^{2}}{4}, \quad y \geqslant u_{+}+2 A^{+}, \\
A^{+}\left[y-\left(A^{+}+u_{+}\right)\right], \\
\quad\left(A^{+}+u_{+}\right)<y \leqslant u_{+}+2 A^{+},
\end{array}\right.
$$

for each $A^{+}>0$. Each case will have to be tackled in turn one at a time.

(a) Recall $n_{0}^{\star}(y)$ was given in (11). In region IV* this given us

$$
v(y, t)=u_{+}+\exp \left(-\frac{\left(y-u_{+}\right)^{2}}{4} t-\frac{1}{2} \ln t-H_{11}(y)+o(1)\right)
$$

as $t \rightarrow \infty$ with $y=O(1)(>0)$ and where the function $H_{11}(y)$ is indeterminate. Nevertheless, comparing with region $\mathrm{III}^{\star}$, we have

$$
H_{11}(y) \sim \ln y-\ln \mathcal{A} \quad \text { as } \quad y \rightarrow \infty .
$$

First the case $-u_{b}<u_{+}<u_{b}$ with $u_{b}>0$ and later on we must think the subcases such as $0<u_{+}<u_{b}$, $u_{+}=0$ and $-u_{b}<u_{+}<0$ distinctly. 
(i) $0<u_{+}<u_{b}$. We note that equation (13) is reversible as $y \rightarrow u_{+}{ }^{+}\left(\in\left(0, u_{b}\right)\right)$ so we refer to a new region as region $\mathrm{A} 1^{\star}$,

$$
w\left(\eta_{\star}\right)=\frac{2 e^{-\eta_{\star}{ }^{2} / 4}}{D_{2}-\sqrt{\pi} \operatorname{erf}\left(\eta_{\star} / 2\right)},
$$

where $D_{2}\left(>\sqrt{\pi},\left(-\infty<\eta_{\star}<\infty\right)\right.$ since requiring $w\left(\eta_{*}\right)>0$ in $\left.\eta_{\star}>0\right)$ is a

constant and $D_{2}=\frac{2}{B}+\sqrt{\pi}$. As $\eta_{\star} \rightarrow-\infty$ moving out of region A1* into region $\mathrm{V}^{\star}$ where $y=O(1)(\epsilon$ $\left.\left(0, u_{+}\right)\right)$. The equation in region $\mathrm{V}^{\star}$ has already been found and is given by

$$
v(y, t)=u_{+}+\exp \left(-\frac{\left(y-u_{+}\right)^{2}}{4} t-\frac{1}{2} \ln t-H_{22}(y)+o(1)\right)
$$

as $t \rightarrow \infty$ with $y=O(1)\left(\in\left(0, u_{+}\right)\right)$, and where the function $H_{22}(y)$ stays indeterminate. Comparing this with region $\mathrm{A} 1^{\star}$ needs that

$$
H_{22}(y) \sim \ln \frac{2}{D_{2}+\sqrt{\pi}} \quad \text { as } \quad y \rightarrow u_{+}^{-} .
$$

To satisfy boundary condition (2) we require equation (13) to be reversible as $y \rightarrow 0^{+}$. To criticize this possible action we refer to a new region as region SS ${ }^{\star}$, where $u=O(1)$ and $y=o(1)$ as $t \rightarrow \infty$.

(ii) $u_{+}=0$. In this case equation (13) is reversible as $y \rightarrow 0^{+}$and to continue the large t-asymptotic schema of $\mathrm{QP}$ we refer to a new region as region $\mathrm{A} 2$, $^{\star}$

$$
w\left(\eta_{\star}\right)=\frac{2 e^{-\eta_{*}{ }^{2} / 4}}{D_{2}^{*}-\sqrt{\pi} \operatorname{erf}\left(\eta_{\star} / 2\right)}, \quad 0 \leqslant \eta_{\star}<\infty,
$$

where $D_{2}^{\star}\left(>\sqrt{\pi}\right.$, since requiring $w\left(\eta_{\star}\right)>0$ in $\left.\eta_{\star}>0\right)$ is a constant. Equation (13) must be reversible as $y \rightarrow 0^{+}$. In region $\mathrm{SS}^{\star}$ we have an equation of the form

$$
v=V(x)+o(1)
$$

as $t \rightarrow \infty$ with $x=O(1)(\geqslant 0)$. On substituting (14) into (1) the leading order problem is found to be

$$
\begin{gathered}
V_{x x}-V V_{x}=0, \quad x>0 \\
V(0)=u_{b}(>0) \\
V(x) \rightarrow u_{+}(<0) \text { as } \quad x \rightarrow \infty .
\end{gathered}
$$

(b) If $n_{0}^{\star}(y)$ is taken as in the appearance (12) then in region $\operatorname{IV}(\mathrm{a})^{\star}$ we obtain

$$
v(y, t)=u_{+}+\exp \left\{-\frac{\left(y-u_{+}\right)^{2}}{4} t-\frac{1}{2} \ln t-H_{R R}(y)+o(1)\right\}
$$

as $t \rightarrow \infty$ with $y=O(1)\left(\in\left(u_{b}, \infty\right)\right)$. In region $\mathrm{TR}^{\star}$

$$
v(\eta, t)=u_{+}+\left(\frac{\left(u_{b}-u_{+}\right)}{2} \operatorname{erfc}\left(\frac{\eta}{2}\right)+o(1)\right) e^{-A^{+2} t-A^{+} \eta t^{1 / 2}}
$$

as $t \rightarrow \infty$ with $\eta=\left(y-u_{b}\right) t^{1 / 2}=O(1)$ and where $A^{+}=\frac{u_{b}-u_{+}}{2}$. In region $\operatorname{IV}(\mathrm{b})^{\star}$

$$
v(y, t)=u_{+}+\exp \left\{-A^{+}[y-c] t+\ln \left(u_{b}-u_{+}\right)\right\}+t^{-1 / 2} H_{L L}(y) \exp \left\{-\frac{\left(y-u_{+}\right)^{2}}{4} t\right\}+o\left(t^{-1 / 2} \exp \left\{-\frac{\left(y-u_{+}\right)^{2}}{4} t\right\}\right)
$$

as $t \rightarrow \infty$ with $y=O(1)\left(\in\left(c, u_{b}\right)\right)$ where $c=\frac{u_{b}+u_{+}}{2}$ and $A^{+}=\frac{u_{b}-u_{+}}{2}$. The function $H_{L L}(y)$ is still indeterminate but by comparing it to region $\mathrm{TR}^{\star}$ as $y \rightarrow u_{b}^{-}$we find

$$
H_{L L}(y) \sim \frac{\mathcal{A}}{2\left(u_{b}-y\right)} \quad \text { as } \quad y \rightarrow u_{b}^{-} .
$$


In region $\mathrm{TW}^{\star}$

$$
v(z, t)=\frac{u_{+}+u_{b} e^{-A^{+} z}}{1+e^{-A^{+} z}}+O\left(t^{-3 / 2} e^{-\frac{A^{+2}}{4} t}\right)
$$

as $t \rightarrow \infty$ with $z=O(1), A^{+}=\frac{u_{b}-u_{+}}{2}, z=\chi-s(t)$

$$
s(t)=\frac{u_{b}+u_{+}}{2} t+O\left(t^{-3 / 2} e^{-\frac{A^{+2}}{4} t}\right)
$$

as $t \rightarrow \infty$. As $z \rightarrow-\infty$ we wave out of region $\mathrm{TW}^{\star}$ into region $\mathrm{V}$ where $y=O(1)=[\in(0, c)]$. There are three different cases to consider.

(i) $-u_{b}<u_{+}<0$. In this situation we have

$$
v(y, t)=u_{b}-\exp \left\{A^{+}[y-c] t+\ln \left(u_{b}-u_{+}\right)+o(1)\right\}
$$

as $t \rightarrow \infty$ with $y=O(1)=(\in(0, c))$. Equation (15) is reversible when $y=O\left(t^{-1}\right)$ [that is, when $x=O(1)$ ] and I refer to a final region $B^{\star}$. In region $B^{\star}$

$$
v(x, t)=u_{b}-\left[\left(u_{b}-u_{+}\right)\left(e^{A^{+} x}-e^{c x}\right)+o(1)\right] e^{-A^{+} c t}
$$

as $t \rightarrow \infty$ for $x=O(1)(\geqslant 0)$.

(ii) $u_{+}=0$. In this situation we first note that $A^{+}=c=\frac{u_{b}}{2}$ so that in region $\mathrm{V}^{\star}$ we have

$$
v(y, t)=u_{b}-\exp \left\{\frac{u_{b}}{2}\left(y-\frac{u_{b}}{2}\right) t+\ln \left(u_{b}-u_{+}\right)+o(1)\right\}
$$

as $t \rightarrow \infty$ with $y=O(1)\left(\in\left(0, \frac{u_{b}}{2}\right)\right)$. In region $\mathrm{B}^{\star}$

$$
v(\xi, t)=u_{b}-\left(u_{b} \operatorname{erf}\left(\frac{\xi}{2}\right)+o(1)\right) e^{-\frac{u_{b}^{2}}{4} t+\frac{u_{b}}{2} \xi t^{1 / 2}}
$$

as $t \rightarrow \infty$ with $\xi=O(1)(\geqslant 0)$.

(iii) $0<u_{+}<u_{b}$. This time region $\mathrm{V}^{\star}$ has to be divided into three regions: region $\mathrm{V}(\mathrm{a})^{\star}\left(0<y<u_{+}\right)$, region $\mathrm{TR}^{\star}$ (transition region) and region $\mathrm{V}(\mathrm{b})^{\star}\left(u_{+}<y<c\right)$. In region $\mathrm{V}(\mathrm{b})^{\star}$

$$
v(y, t)=u_{b}-\exp \left\{-\frac{\left(y-u_{b}\right)^{2}}{4} t-\frac{1}{2} \ln t-H_{p p}(y)+o(1)\right\}
$$

as $t \rightarrow \infty$ with $y=O(1)\left(\in\left(0, u_{+}\right)\right)$. The function $H_{p p}(y)$ stays indeterminate, but matching as $y \rightarrow 0$ requires that

$$
H_{p p}(y) \sim-\ln y+\beta \star
$$

as $y \rightarrow 0^{+}$. In region $\mathrm{B}^{\star}$

$$
v(x, t)=u_{b}-\left(e^{-\beta_{x}} x e^{\frac{u_{b}}{2} x}+o(1)\right) t^{-3 / 2} e^{-\frac{u_{b}^{2}}{4} t}
$$

as $t \rightarrow \infty$ for $x=O(1)(\geqslant 0)$. This then completes the asymptotic schema in this case.

\section{Asymptotic schema when $-\mathbf{u}_{+}>\mathbf{u}_{\mathbf{b}}>\mathbf{u}_{+}, \mathbf{u}_{+}<0$}

The asymptotic schema of QP as $t \rightarrow 0$ and as $x \rightarrow \infty(t=O(1)(>0))$ in this section is followed directly like in section 2 and is not repeated here. In region $\operatorname{IV}(\mathrm{a})^{\star}$

$$
v(y, t)=u_{+}+\exp \left\{-\frac{\left(y-u_{+}\right)^{2}}{4} t-\frac{1}{2} \ln t-H_{R R}(y)+o(1)\right\}
$$


as $t \rightarrow \infty$ with $y=O(1)\left(\in\left(-u_{+}, \infty\right)\right)$ and $H_{R R}(y)$ being still indeterminate but matching with region III* in section 2 that

$$
H_{R R}(y) \sim\left\{\begin{array}{l}
\ln y-\ln \mathcal{A}^{+} \text {as } \quad y \rightarrow \infty, \\
\ln \left(y+u_{+}\right)+\beta_{1} \quad \text { as } \quad y \rightarrow\left(-u_{+}\right)^{+}
\end{array}\right.
$$

where $\beta_{1}=E_{R R}+\ln \sqrt{\pi}$ and $E_{R R}$ needs to be identified. In region $\mathrm{TR}^{\star}$

$$
v(\eta, t)=u_{+}+\left(\frac{1}{2} e^{-E_{R R}} \operatorname{erfc}\left(\frac{\eta_{\star}}{2}\right)+o(1)\right) e^{-u_{+}^{2} t+u_{+} \eta_{\star} t^{1 / 2}}
$$

as $t \rightarrow \infty$ with $\eta_{\star}=\left[y+u_{+}\right] t^{1 / 2}=O(1)$. In region $\mathrm{IV}(\mathrm{b})^{\star}$

$$
v(y, t)=u_{+}+\exp \left\{u_{+} y t-E_{R R}+o(1)\right\}
$$

as $t \rightarrow \infty$ with $y=O(1)\left(\in\left(0,-u_{+}\right)\right)$. In region $\mathrm{V}^{\star}$

$$
v(x, t)=u_{+} \tanh \left(-\frac{u_{+}}{2} x+\tanh ^{-1}\left(\frac{u_{b}}{u_{+}}\right)\right)+o(1)
$$

as $t \rightarrow \infty$ with $x \geqslant 0$. The schema in this case is now completed.

\section{Asymptotic schema while $\mathbf{u}_{\mathrm{b}}<\mathbf{u}_{+}<0, \mathbf{u}_{\mathrm{b}}<0$}

The large t-asymptotic schema in this case follows closely that summarized in Section 3. In region IV(a)*

$$
v(y, t)=u_{+}-\exp \left\{-\frac{\left(y-u_{+}\right)^{2}}{4} t-\frac{1}{2} \ln t-H_{R R}(y)+o(1)\right\}
$$

as $t \rightarrow \infty$ with $y=O(1)\left(\in\left(-u_{+}, \infty\right)\right)$ and $H_{R R}(y)$ stays indeterminate but matching with region III $^{\star}$ in section 2 requires

$$
H_{R R}(y) \sim\left\{\begin{array}{l}
\ln y-\ln \mathcal{A} \quad \text { as } \quad y \rightarrow \infty, \\
\ln \left(y+u_{+}\right)+\beta_{1} \quad \text { as } \quad y \rightarrow\left(-u_{+}\right)^{+}
\end{array}\right.
$$

where $\beta_{1}=E_{R R}+\ln \sqrt{\pi}, \mathcal{A}=\frac{2}{\sqrt{\pi}}\left(u_{+}-u_{b}\right)(>0)$ and $E_{R R}$ is a constant. In region $\mathrm{TR}^{\star}$

$$
v\left(\eta_{\star}, t\right)=u_{+}-\left(\frac{1}{2} e^{-E_{R R}} \operatorname{erfc}\left(\frac{\eta_{\star}}{2}\right)+o(1)\right) e^{-u_{+}^{2} t+u_{+} \eta_{*} t^{1 / 2}}
$$

as $t \rightarrow \infty$ with $\eta_{\star}=\left[y+u_{+}\right] t^{1 / 2}=O(1)$. In region $\operatorname{IV}(\mathrm{b})^{\star}$

$$
v(y, t)=u_{+}-\exp \left\{u_{+} y t-E_{R R}+o(1)\right\}
$$

as $t \rightarrow \infty$ with $y=O(1)\left(\in\left(0,-u_{+}\right)\right)$. In region $\mathrm{V}^{\star}$

$$
v(x, t)=u_{+} \operatorname{coth}\left(-\frac{u_{+}}{2} x+\operatorname{coth}^{-1}\left(\frac{u_{b}}{u_{+}}\right)\right)+o(1),
$$

as $t \rightarrow \infty$ with $x \geqslant 0$.

\section{Asymptotic schema when $\mathbf{u}_{\mathrm{b}}<0, \mathbf{u}_{+}=0$}

The asymptotic solution of QP as $t \rightarrow \infty$ differs from that seen in Sections 3 and 4. In region IV*

$$
v(y, t)=-\exp \left\{-\frac{y^{2}}{4} t-\frac{1}{2} \ln t-H_{R R}(y)+o(1)\right\}
$$


as $t \rightarrow \infty$ with $y=O(1)(\in(0, \infty))$ and $H_{R R}(y)$ is not determined but matching with region III $^{\star}$ in section 2 requires

$$
H_{R R}(y) \sim \begin{cases}\ln y-\ln \mathcal{A} & \text { as } \quad y \rightarrow \infty, \\ -\ln \mathcal{C} \text { as } \quad y \rightarrow 0^{+},\end{cases}
$$

where $\mathcal{A}=\frac{2\left(-u_{b}\right)}{\sqrt{\pi}}(>0)$ in this case. As $y \rightarrow 0$ this equation is reversible and we refer to a new region as region $\mathrm{V}^{\star}$. Therefore, in region $\mathrm{V}^{\star}$

$$
V(\xi, t)=-\frac{2}{\sqrt{\pi}} \frac{e^{-\xi^{2} / 4}}{\operatorname{erf}\left(\frac{\xi}{2}\right)} t^{-1 / 2}+o\left(t^{-1 / 2}\right)
$$

as $t \rightarrow \infty$ with $\xi=O(1)(>0)$. we desire the equation be reversible as $\xi \rightarrow 0^{+}$. A new region is needed; in region $\mathrm{VI}^{\star}$

$$
v(x, t)=\frac{2}{\frac{2}{u_{b}}-x}+o(1)
$$

as $t \rightarrow \infty$ with $x=O(1)(\geqslant 0)$.

\section{Asymptotic schema while $\mathbf{u}_{+}>\mathbf{u}_{\mathrm{b}} \geqslant 0$}

In region $\mathrm{IV}^{\star}$

$$
v(y, t)=u_{+}-\exp \left\{-\frac{\left(y-u_{+}\right)^{2}}{4} t-\frac{1}{2} \ln t-H_{R R}(y)+o(1)\right\}
$$

as $t \rightarrow \infty$ with $y=O(1)(\in(0, \infty))$ and $H_{R R}(y)$ is indeterminate but matching with region III $^{\star}$ in section 2 requires that

$$
H_{R R}(y) \sim\left\{\begin{array}{l}
\ln y-\ln \mathcal{A} \quad \text { as } \quad y \rightarrow \infty, \\
-\ln \frac{1}{\sqrt{\pi}} \quad \text { as } \quad y \rightarrow u_{+}^{+},
\end{array}\right.
$$

so $\mathcal{A}=\frac{2\left(u_{+}-u_{b}\right)}{\sqrt{\pi}}(>0)$ in this case. We need to consider the subcases:

(i) $u_{+}>u_{b}>0$,

and

(ii) $u_{b}=0$,

separately. We start with the first subcase

(i) $u_{+}>u_{b}>0$. In region $\mathrm{A}$ *

$$
v\left(\eta_{\star}, t\right)=u_{+}-\frac{2 e^{-\eta_{\star}{ }^{2} / 4}}{\sqrt{\pi} \operatorname{erfc}\left(\eta_{\star} / 2\right)} t^{-1 / 2}+o\left(t^{-1 / 2}\right)
$$

as $t \rightarrow \infty$ with $\eta_{\star}=O(1)$. As $\eta_{\star} \rightarrow-\infty$ moving out from region $\mathrm{A}^{\star}$ into region $\mathrm{V}^{\star}$. In region $\mathrm{V}^{\star}$

$$
v(y, t)=y+o(1)
$$

as $t \rightarrow \infty$ with $y=O(1)\left(\in\left(u_{b}, u_{+}\right)\right)$. We note that this equation is reversible as $y \rightarrow u_{b}^{+}$and we must refer to another one that is called region $\mathrm{B}^{\star}$, located at $y=u_{b}$. In region $\mathrm{B}$ we have

$$
v\left(\eta_{*}, t\right)=u_{b}+\frac{2 e^{-\frac{\eta_{*}^{2}}{4}}}{\sqrt{\pi}\left(1-\operatorname{erf}\left(\frac{\eta_{*}}{2}\right)\right)} t^{-\frac{1}{2}}+o\left(t^{-\frac{1}{2}}\right)
$$

as $t \rightarrow \infty$ with $\eta_{\star}=O(1)$. As $\eta_{\star} \rightarrow-\infty$ from the localised region $\mathrm{B}^{\star}$ into region $\mathrm{VI}^{\star}$,

$$
v(y, t)=u_{b}+\exp \left(-\frac{\left(y-u_{b}\right)^{2}}{4} t-\frac{1}{2} \ln t-L L(y)+o(1)\right)
$$


as $t \rightarrow \infty$ with $y=O(1)\left(\in\left(0, u_{b}\right)\right)$ and $L L(y)$ is not determined, but matching region III ${ }^{\star}$ in section 2 requires

$$
L L(y) \sim \frac{1}{\sqrt{\pi}} \quad \text { as } \quad y \rightarrow u_{b}^{-} .
$$

We conclude that the equation must be reversible as $y \rightarrow 0^{+}$. We introduce a final asymptotic region, in region $\mathrm{C}^{\star}$.

$$
v(x, t)=u_{b}+x e^{-\beta_{\star}} e^{\frac{u_{b}}{2} x} t^{-3 / 2} e^{-\frac{u_{b}^{2}}{4} t}+o\left(t^{-3 / 2} e^{-\frac{u_{b}^{2}}{4} t}\right)
$$

as $t \rightarrow \infty$ with $x=O(1)$.

(ii) $u_{b}=0$. In this subcase the asymptotic schema of the solution of QP given in regions $\mathrm{IV}^{\star}, \mathrm{A}^{\star}$ and $\mathrm{V}^{\star}$ follows on setting $A=\frac{2 u_{+}}{\sqrt{\pi}}$ and $u_{b}=0$ that given above after some calculation region $\mathrm{C}^{\star}$ has

$$
v(x, t)=x t^{-1}+o\left(t^{-1}\right)
$$

as $t \rightarrow \infty$ with $x=O(1)$. Now the asymptotic schema of solution to QP is complete as $t \rightarrow \infty$.

\section{Asymptotic schema while $\mathbf{u}_{\mathrm{b}}<0 \quad \mathbf{u}_{+}>0$}

The difference from Section 6 is that region $\mathrm{B}^{\star}$ has,

$$
v\left(\eta_{\star}, t\right)=\left(\eta-\frac{2}{\eta_{\star}}\right) t^{-1 / 2}+o\left(t^{1 / 2}\right)
$$

as $t \rightarrow \infty$ with $\eta_{\star}=O(1)(>0)$. In region $\mathrm{SS}^{\star}$

$$
v(x, t)=\frac{2}{\frac{2}{u_{b}}-x}+o(1)
$$

as $t \rightarrow \infty$ with $x=O(1)(\geqslant 0)$.

\section{Numerical solutions}

Here we use the numerical method summarized in [10] to solve QP. We consider numerical simulation of QP for each case in turn:

(i) $-u_{b}<u_{+}<u_{b}$ with $u_{b}>0$.

It appears as though the numerical solution overlaps quickly to the awaited TW where we assume the overlap to be exponential in $t$ as $t \rightarrow \infty$. 


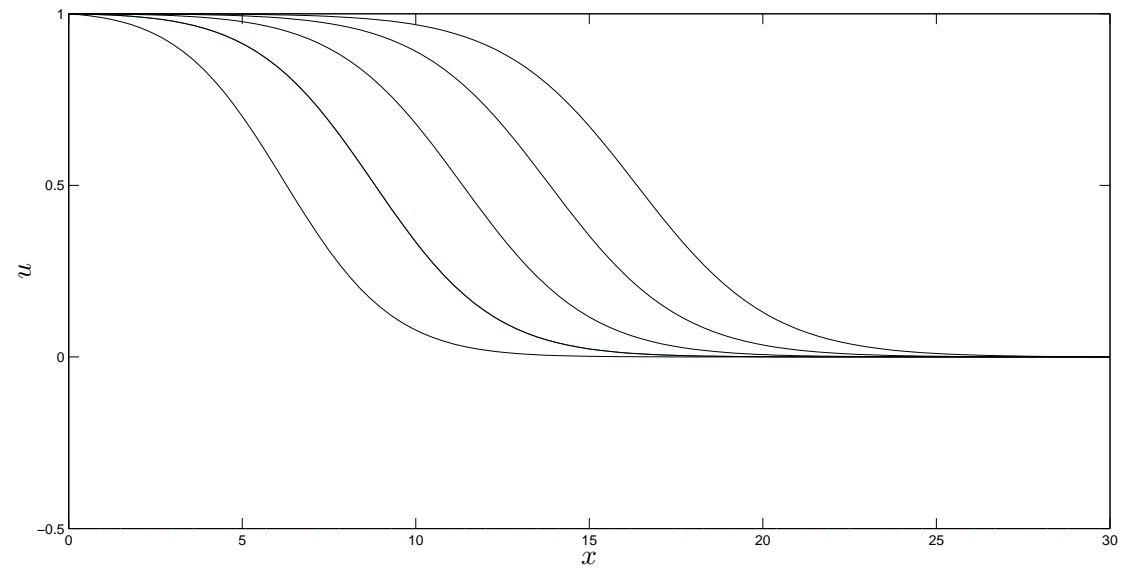

Figure 1: QP is numerically solved for $u_{b}=1$ and $u_{+}=0$ at times $t=5,10,15,20$ and $t=25$.

Figure 2 shows the quick overlap of the numerically computed ws to the theoretically predicted ws which is again in agreement with the theory.

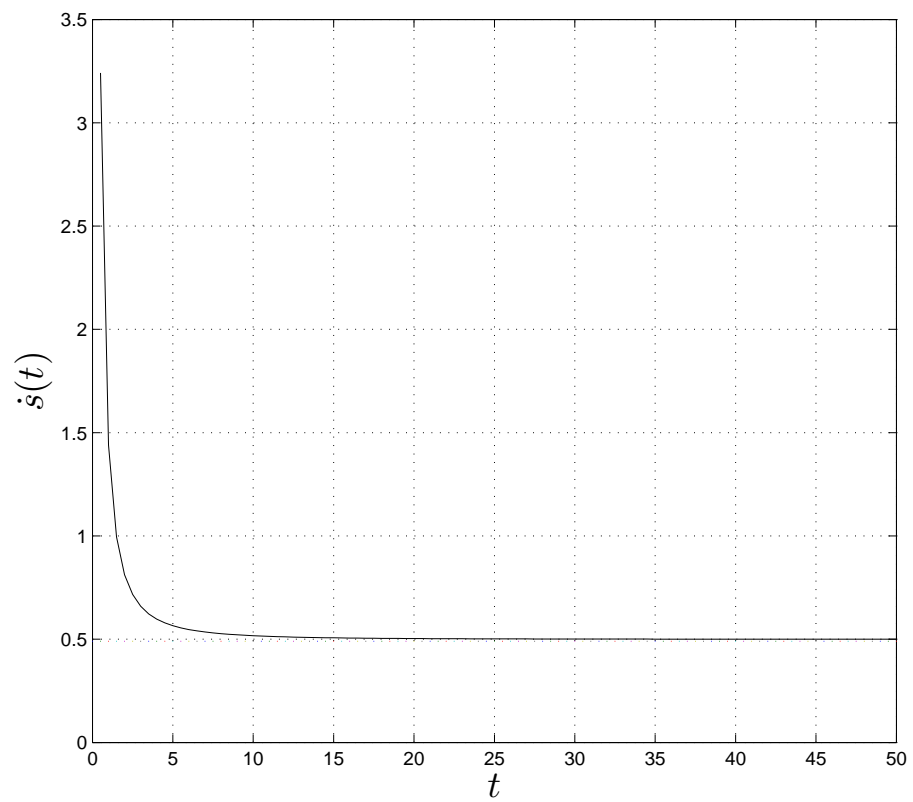

Figure 2: Numerical solution of $\dot{s}(t)$ versus $t$. 
(ii) $-u_{+}>u_{b}>u_{+}$with $u_{+}<0$.

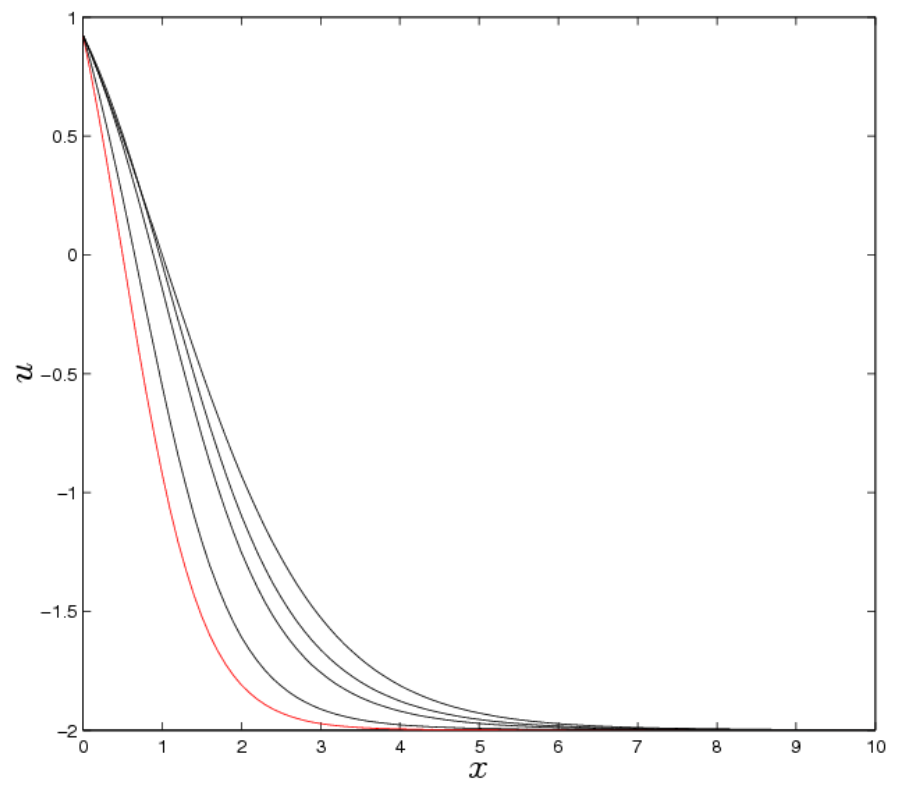

Figure 3: Graph of the numerical solution of QP in the $(x, u=v)$ plane for $u_{b}=1$ and $u_{+}=-2$ at times $t=5,10,15,20$ and 25. We note that the exact SS is shown via the red line at $t=25$.

(iii) $u_{b}<u_{+}<0, u_{b}<0$.

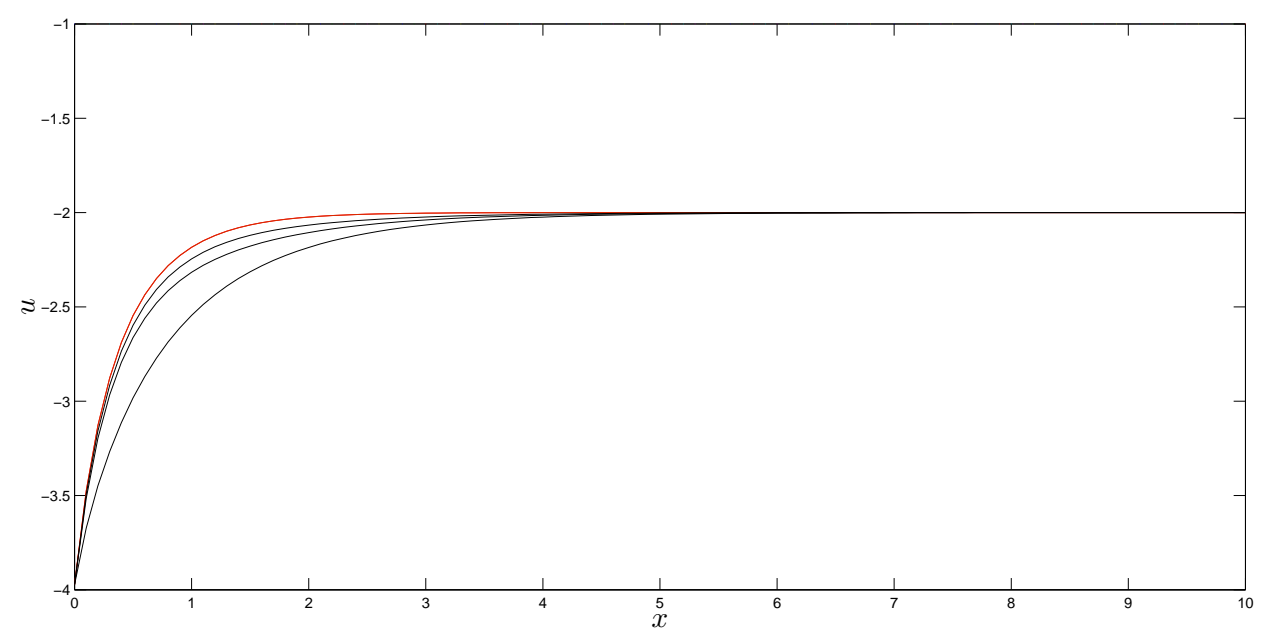

Figure 4: Graph of the numerical solution of QP in the $(x, u=v)$ plane for $u_{b}=-2$ and $u_{+}=-1$ at times $t=5,10,15$ and 20 . We note that the exact SS is shown via the red line at $t=20$. 
(iv) $u_{b}<0$ and $u_{+}=0$.

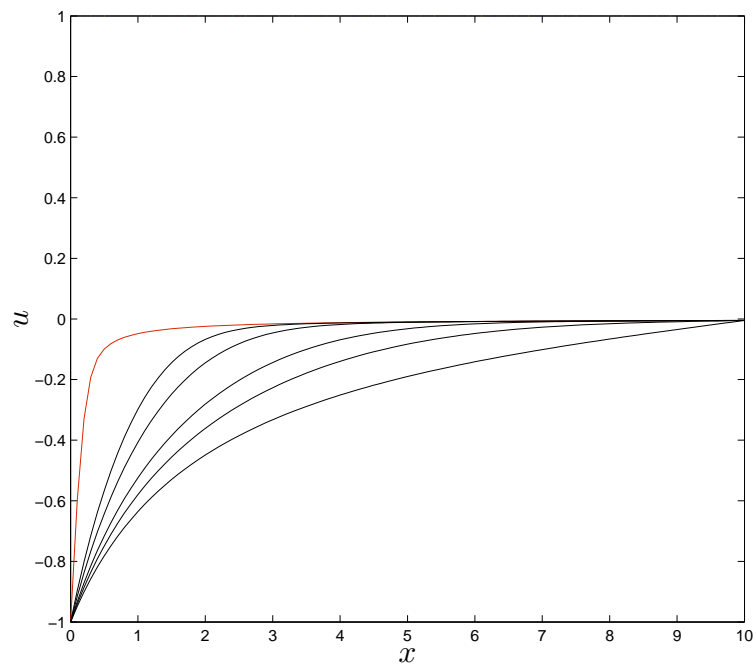

Figure 5: Graph of the numerical solution of QP in the $(x, u=v)$ plane for $u_{b}=-1$ and $u_{+}=0$ at times $t=5,10,15,20,25$ and 30 . We note that the exact SS is shown via the red line at $t>30$.

(v) $u_{+}>u_{b} \geqslant 0$.

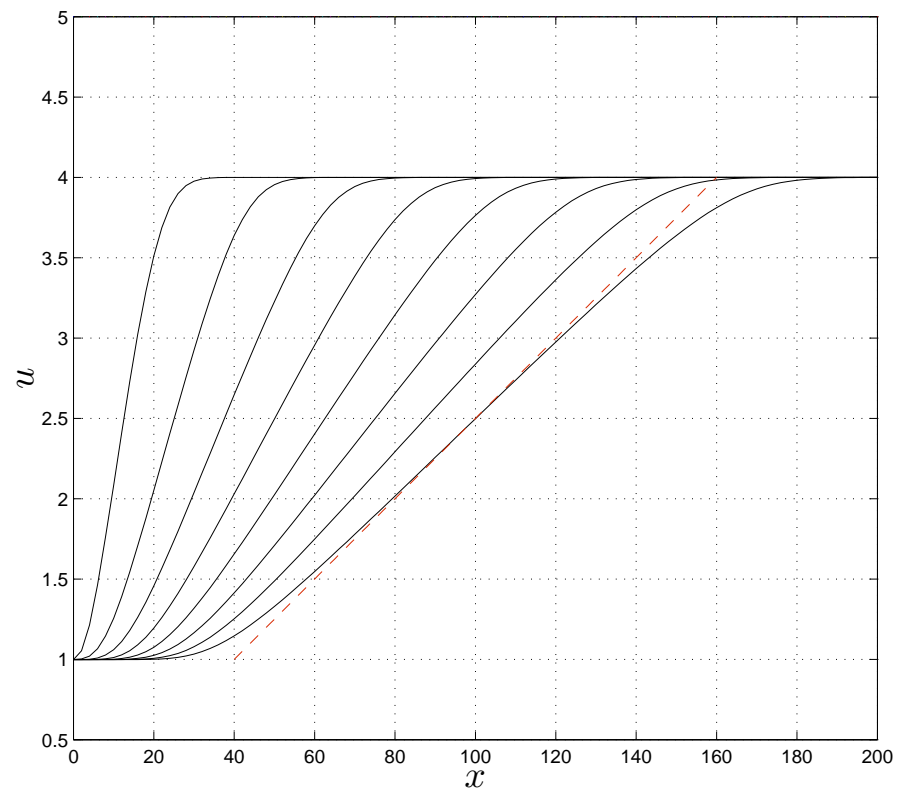

Figure 6: The occurrence of the EW with the solid lines showing the numerically computed solutions and the red dash line representing the predicted slope. 
(vi) $u_{b}<0$ and $u_{+}>0$.

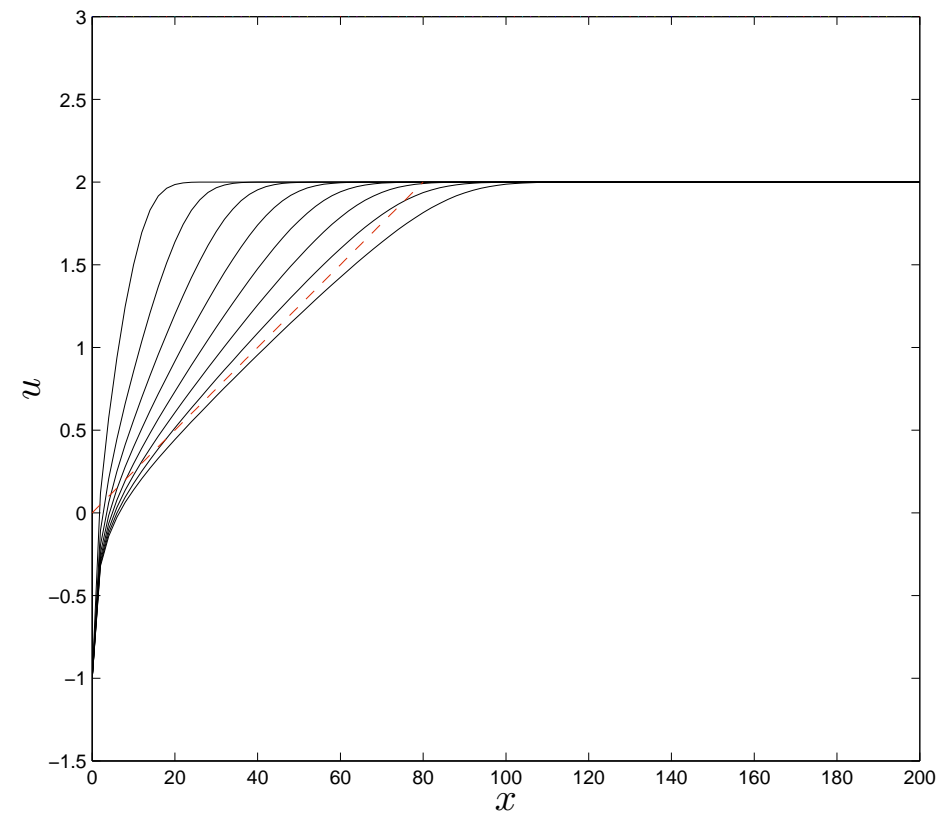

Figure 7: The occurrence of EW and SS with the solid lines show numerically computed solutions and the dash line representing the predicted slope at $t=40$.

Further, it is easy to see there is an overlap among the numerical solution and the stationary state while $u_{b}=-1$ given by

$$
v(x)=-\frac{2}{x-2},
$$

over the range $x \in\left[0, b t^{1 / 2}\right]$ where $b$ is positive fixed number.

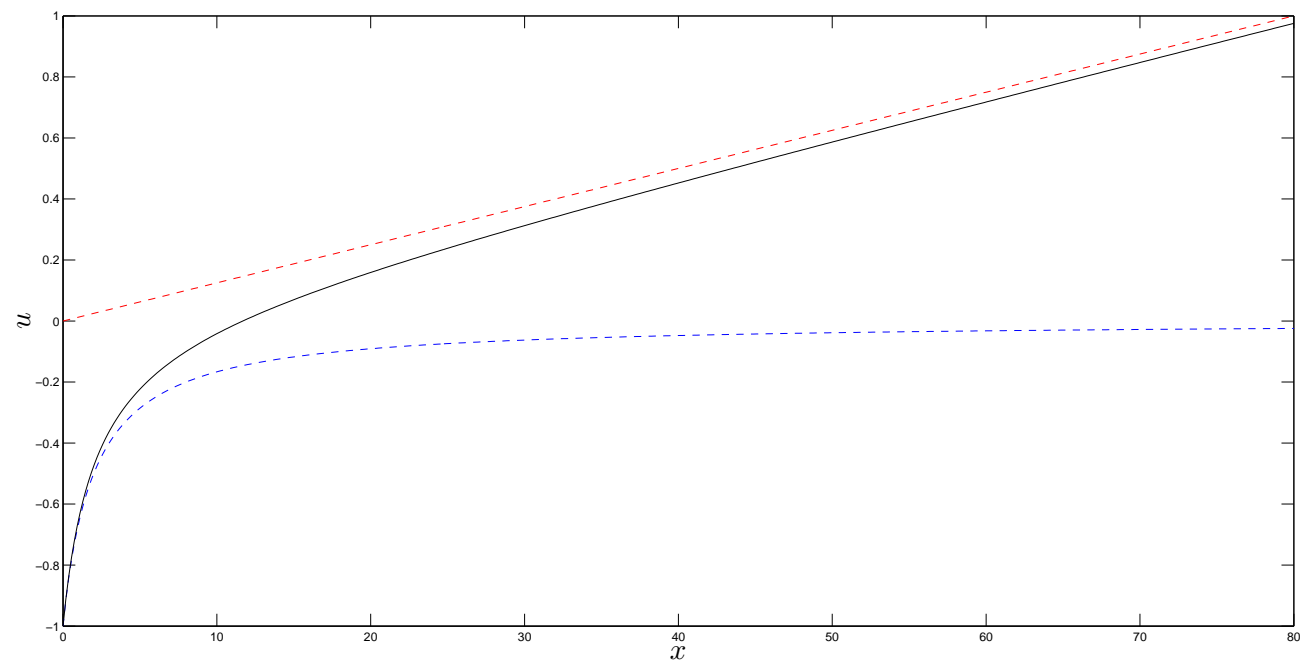

Figure 8: The numerical solution of QP in the $(x, u=v)$ for $x \in[0,80]$ for $u_{b}=-1$ and $t=40$. 


\section{Conclusion}

The entire asymptotic schema of QP have been obtained as $t \rightarrow \infty$ over whole parameter measures. The type of large t-attractor that occurs as $t \rightarrow \infty$ is controlled by $u_{+}$and $u_{b}$. In the second Section a TW evolved as $t \rightarrow \infty$ in the QP. The grade of overlap of the QP onto the TW is exponential in $t$, being of $O\left(t^{-3 / 2} e^{-\frac{A^{2}}{4} t}\right)$ as $t \rightarrow \infty$. In the third section it is important to note that the grade of overlap of the QP is exponential in $t$ as $t \rightarrow \infty$. In the fourth section, as in third section, the grade of overlap of the QP is exponential in $t$ as $t \rightarrow \infty$ has been obtained. In the section follwing section 4 and throughout the regions an irreversible approximation was obtained. In section 6 the solution displayed the appearance of an EW for $x \geqslant 0$ and where $u_{+}>u_{b} \geqslant 0$. In Section 7, as in Section 5, the same irreversible approximation was found throughout the regions. In Section 8, numerical solutions of QP are represented that affirm and support the asymptotic analysis presented in the sections mentioned above. In all case the numerical simulations are in good agreement with the theory as $t \rightarrow \infty$. In conclusion, Equation (1)-(3) arises in mathematical chemistry, biology, physics and play a vital role in related areas.

\section{References}

[1] Aronson D. G., Weinberger H. F., Multidimensional nonlinear diffusion arising in propagation genetics, Adv. Math., 1978, 30(1), 33-76

[2] Aronson D. G., Weinberger H. F., Nonlinear diffusion in population genetics, combustion, and nerve pulse propagation, In: Goldstein J. A. (Ed.), Partial Differential Equations and Related Topics, Springer Berlin, Lecture Notes in Mathematics, 1975, 446, 5-49

[3] Bramson M. D., Maximal displacement of branching Brownian motion, Comm. Pure Appl. Math., 1978, 31, 531-582

[4] Burgers J. M., A mathematical model illustrating the theory of turbulence, Adv. Appl. Mech., 1948, 1, 171-199

[5] Burgers J. M., Mathematical examples illustrating relations occurring in the theory of turbulent fluid motion, Kon. Ned. Akad. Wet., Verh. (Eerste Sectie), 1939, 17(2), 1-53

[6] Hanaç E., The large-time solution of nonlinear evolution equations, Ph. D. Thesis, University of Birmingham, 2015

[7] Leach J. A., Needham D. J., Matched asymptotic expansions in reaction-diffusion theory, Springer Monographs in Mathematics, Springer-Verlag London, 2004

[8] Leach J. A., Hanaç E., On the evolution of travelling wave solutions of the Burgers-Fisher equation, Quart. Appl. Math., 2016, 74, 337-359

[9] Hanaç E., The phase plane analysis of nonlinear equation, J. Math. Anal., 2018, 9, 89-97

[10] Landejuela M., Burgers equation, BCAM Internship report: Basque Center for Applied Mathematics, 2011 